\section{The APC/C Inhibitor XErp1/Emi2 Is Essential for Xenopus Early Embryonic Divisions}

\author{
Thomas Tischer, Eva Hörmanseder, ${ }^{*}$ Thomas U. Mayer†
}

Mitotic divisions result from the oscillating activity of cyclin-dependent kinase 1 (Cdk1). Cdk1 activity is terminated by the anaphase-promoting complex/cyclosome (APC/C), a ubiquitin ligase that targets cyclin B for destruction. In somatic divisions, the early mitotic inhibitor 1 (Emi1) and the spindle assembly checkpoint $(S A C)$ regulate cell cycle progression by inhibiting the APC/C. Early embryonic divisions lack these $A P C / C$-inhibitory components, which raises the question of how those cycles are controlled. We found that the APC/C-inhibitory activity of XErp1 (also known as Emi2) was essential for early divisions in Xenopus embryos. Loss of XErp1 resulted in untimely destruction of $A P C / C$ substrates and embryonic lethality. XErp1's APC/C-inhibitory function was negatively regulated by $C \mathrm{dk} 1$ and positively by protein phosphatase $2 \mathrm{~A}$ (PP2A). Thus, Cdk1 and PP2A operate at the core of early mitotic cell cycles by antagonistically controlling XErp1 activity, which results in oscillating $A P C / C$ activity.

\footnotetext{
A fter fertilization and a prolonged first cell cycle, Xenopus embryos progress through 11 rapid divisions devoid of gap phases. Cycle 13 marks mid-blastula transition (MBT), when cycles become longer and gap phases are resumed $(1-3)$. Although it is clear that cyclin-

Department of Biology and Konstanz Research School Chemical Biology, University of Konstanz, Universitätsstr. 10, 78457 Konstanz, Germany.

"These authors contributed equally to this work.

tro whom correspondence should be addressed. E-mail: thomas.u.mayer@uni-konstanz.de
}

dependent kinase $1(\mathrm{Cdk} \mathrm{I})$ is the universal cell cycle regulator, it remains unknown how pre-MBT divisions lacking inhibitory phosphorylations of $\mathrm{Cdkl}(2,4)$ as well as the anaphase-promoting complex/cyclosome $(\mathrm{APC} / \mathrm{C})$-inhibitory activities of Emil and SAC $(3,5,6)$ are controlled. Before fertilization, XErpl mediates the metaphase II arrest of mature Xenopus eggs by directly inhibiting the APC/C (7). Surprisingly, XErp I is completely degraded at fertilization but reaccumulates in early embryos (8-12), yet egg extract studies suggest that XErpl has no function in mitosis $(9)$.
To understand the regulation of early mitotic divisions, we first examined XErpl levels in Xenopus embryos by immunoblot analyses. After its destruction at fertilization, XErpl reaccumulated to levels comparable to those in unfertilized eggs and started to disappear again at MBT, which is marked by the destruction of cyclin E1 and replacement of cyclin A1 by cyclin A2 (Fig. 1A). To test whether XErpl expression is critical for early mitotic cycles, we injected antisense morpholino-oligos (MO) targeting XEip1 mRNA (XErp1-MO) or control sense MO into one-cell embryos (13). At 24 hours post-fertilization (hpf), $92 \%$ of the control MO-injected embryos displayed small blastopores (Fig. 1, B and C), revealing that these embryos completed the first major morphogenetic transformation (i.e., blastopore closure). In contrast, $90 \%$ of XErp1-depleted embryos failed to undergo blastopore closure but underwent apoptosis at gastrulation (Fig. 1, B to D; fig. S1, A and B; and movie S1). To confirm that loss of XErpl accounted for the observed phenotype, we co-injected embryos with XErpl$\mathrm{MO}$ and wild-type (WT) XErpl mRNA (myc$\mathrm{XErpl}{ }^{\mathrm{WT}}$ ) that was not targeted by the MO (Fig. 1D). Expression of myc-tagged full-length XErpl ${ }^{\text {WT }}$ efficiently rescued blastopore closure in XErp1depleted embryos (Fig. 1, B and C). Thus, XErp1 is essential for Xenopus early embryonic cycles.

Next, we analyzed whether these divisions depend on XErpl's APC/C-inhibitory activity. Indeed, the majority of XErpl-depleted embryos expressing XErpl mutated in its zinc-binding region (ZBR ) or destruction box ( $\triangle$ box ${ }^{-}$) both of 
which are deficient in $\mathrm{APC} / \mathrm{C}$ inhibition $(7, I I)$ (fig. S5)-failed to undergo blastopore closure, whereas Fbox-mutant XErp1, which is not com- promised in $\mathrm{APC} / \mathrm{C}$ inhibition (7), efficiently rescued the loss of XErp1 (Fig. 1, B and C, and fig. $\mathrm{SI}, \mathrm{C}$ to $\mathrm{E}$ ). Consequently, relative to con- trol embryos, XErpl-depleted embryos displayed reduced levels of the APC/C substrate cyclin B2 (Fig. 2A) and increased cell cycle lengths (fig.
A

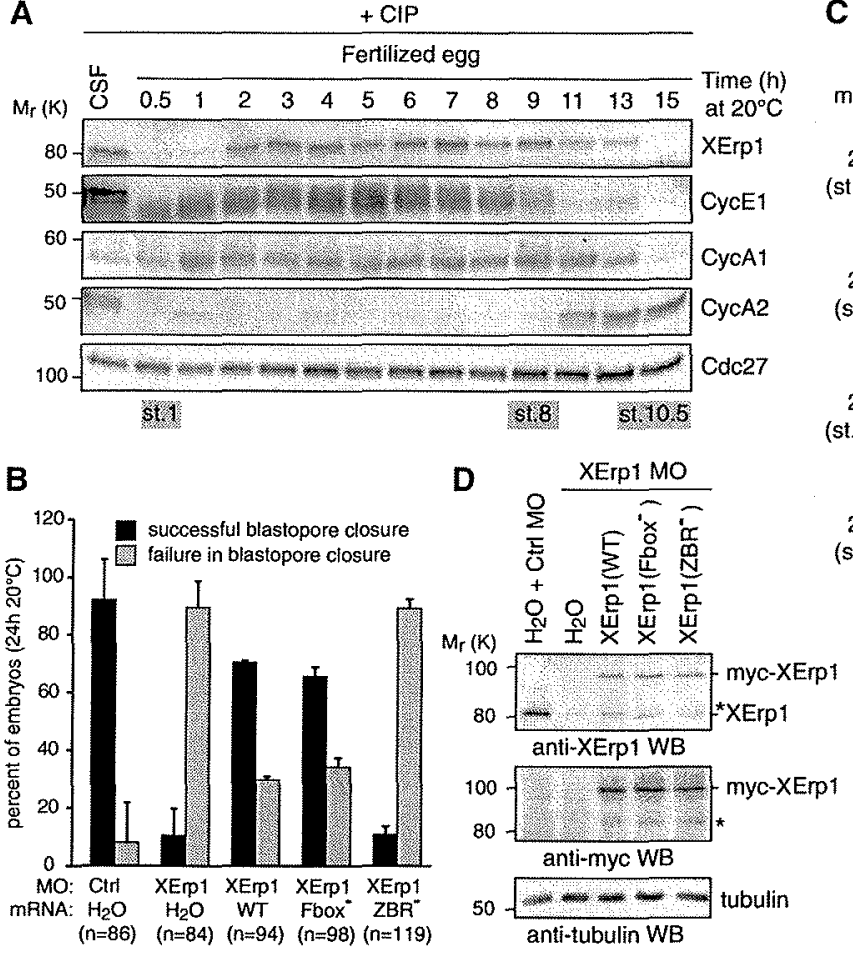

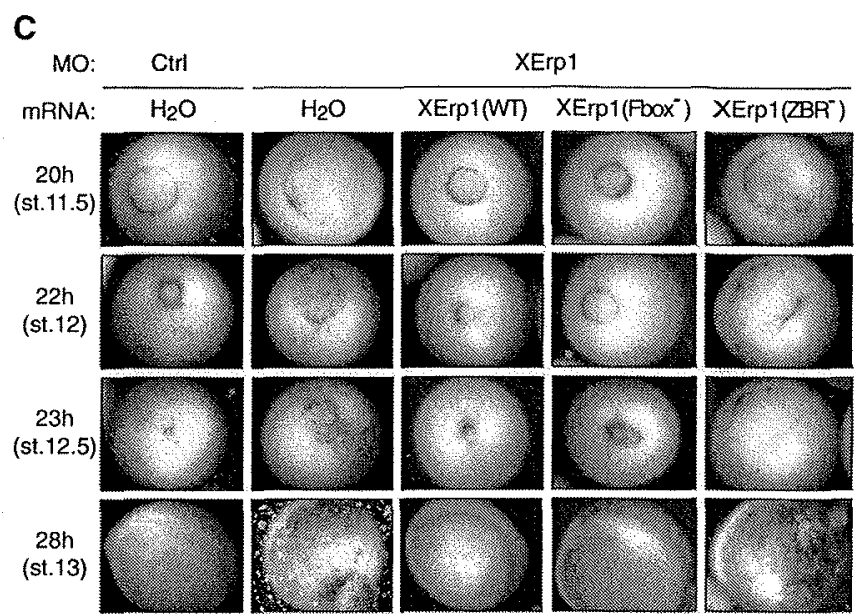

Fig. 1. XErp1 is essential for early embryonic divisions. (A) Immunoblot analyses of calf intestinal phosphatase (CIP)-treated samples harvested at the indicated time points. (B and $\mathbf{C}$ ) One-cell embryos were injected with XErpI-MO or control MO and $\mathrm{H}_{2} \mathrm{O}$ or mRNA encoding WT, Fbox mutant, or ZBR- mutant XErp1; 24-hpf phenotypes were quantified (B) or images were taken at the indicated times (C). (D) Embryos used in (B) were lysed at $6 \mathrm{hpf}$ and immunoblotted. Asterisk marks nonspecific band. Cyc, cyclin.
A

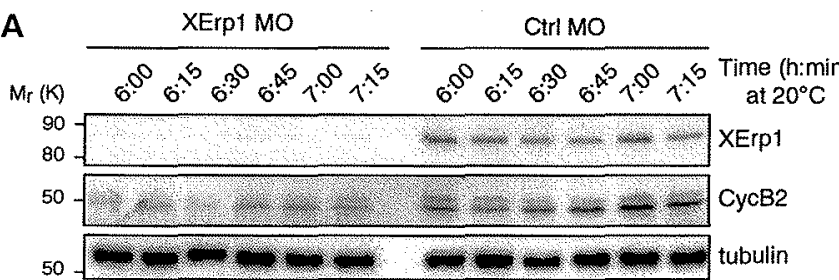

B

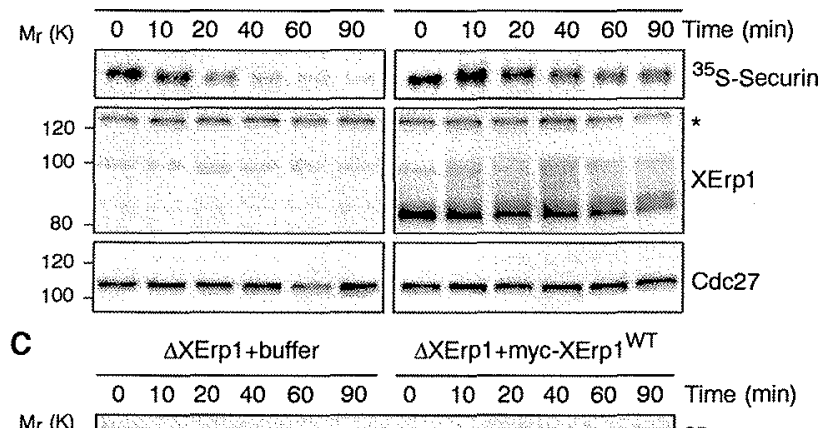

D

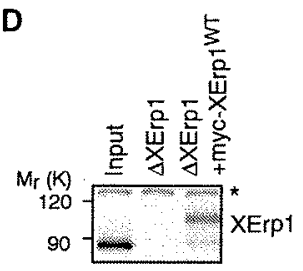

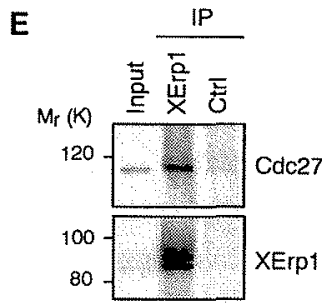

$\mathbf{F}$

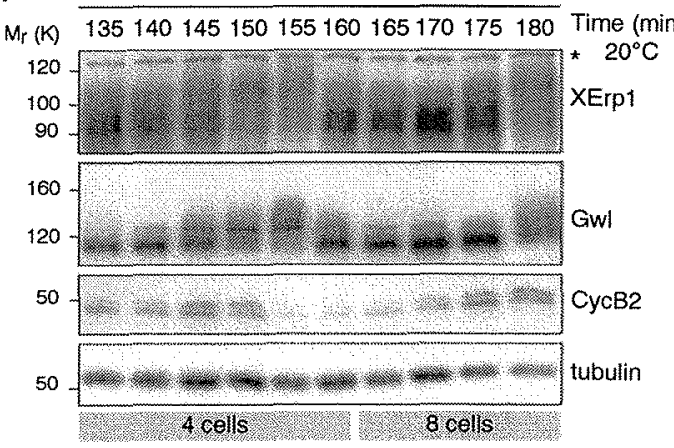

Fig. 2. XErp1 is required for timely destruction of $A P C / C$ substrates. (A) One-cell embryos were injected with control MO (Ctrl) or XErp1-MO, lysed at the indicated time points, and immunoblotted. (B) Embryo extract depleted of XErp1 or control immunoglobulin $G$ extract was supplemented with ${ }^{35} \mathrm{~S}$. labeled securin and at the indicated time points, samples were analyzed by immunoblot and autoradiography analysis. (C) XErp1-depleted extract was supplemented with buffer or IVT myc-XErp1 ${ }^{\text {WT }}$ and samples were analyzed as in (B). (D) Extract from (C) was treated with CIP and immunoblotted for XErp1. Asterisk marks nonspecific band. (E) At 4 hpf, embryos were lysed, and XErp1 and control immunoprecipitates were immunoblotted for XErp1 and Cdc27. (F) Non-CIP-treated embryo lysates were immunoblotted. Gwl, greatwall kinase. 
S2, A and B). To further confirm this finding, we established an embryonic extract system that allowed us to monitor APC/C activity by analyzing the stability of in vitro translated (IVT) ${ }^{35}$ S-labeled securin. Depletion of XErp1 caused $\mathrm{APC} / \mathrm{C}$ activation indicated by the destruction of ${ }^{35} \mathrm{~S}$-labeled securin, and this effect could be rescued by IVT myc-XErpl ${ }^{\text {WT }}$ (Fig. 2, B to D). Consequently, XErp1 immunopurified from embryo extract associated with the APC/C core subunit Cdc27 (Fig. 2E). Thus, the APC/C inhibitor XErpl is critical for timely destruction of APC/C substrates in early Xenopus embryos.

In metaphase II, Cdk1 transiently activates the APC/C to compensate for continuous cyclin B synthesis by phosphorylating XErpl at $\mathrm{Ser}^{213}$, $\mathrm{Thr}^{239}, \mathrm{Thr}^{252}$, Thr $r^{267}$, $\mathrm{Thr}^{545}$, and $\mathrm{Thr}^{551}\left(\mathrm{ST}_{5}\right)$, which decreases its half-life and affinity for the $\operatorname{APC} / \mathrm{C}(14,15)$. Negative regulation by $\mathrm{Cdk} 1$ is antagonized by protein phosphatase 2A (PP2A), which, upon phosphorylation of XErpI by p90Rsk, binds to XErpl and keeps dephosphorylating it $(8,11,14-16)$. Because XErp1 remained at constant levels during early divisions (fig. S3A) but underwent cell cycle-dependent changes in its electrophoretic mobility (Fig. $2 \mathrm{~F}$ and fig. S3B), we speculated that XErpl might be regulated by
Cdk1 and PP2A. If this applies, expression of XErpl mutated at the six Cdkl phosphorylation sites $\left(\mathrm{ST}_{5} \rightarrow 6 \mathrm{~A}\right)$ should cause a cell cycle arrest due to constitutive $\mathrm{APC} / \mathrm{C}$ inhibition. Indeed, 6-hpf XErpI-depleted embryos expressing mycXErpl ${ }^{6 A}$ displayed gigantic cells and elevated cyclin B2 levels (Fig. 3, A and B, and fig. S3, C and $D$ ). This cell cycle arrest was caused by APC/C inhibition, because expression of mycXErpl ${ }^{6 A Z B R-}$ had no effect (Fig. 3, A and B, and fig. $\mathrm{S} 3 \mathrm{C}$ ). To further confirm these findings, we analyzed the stability of APC/C substrates in embryo extract whene we elevated Cdk1 activity by the addition of nondegradable cyclin $\mathrm{B}\left(\mathrm{CycB}^{\Delta 90}\right)$. Intriguingly, $25 \mathrm{nM} \mathrm{CycB}^{\Delta 90}$ was sufficient to induce the degradation of cyclin $\mathrm{B} 2$ and ${ }^{35} \mathrm{~S}$-labeled securin (Fig. 3C). Elevated Cdk1 activity correlated with the phosphorylation of XErp1 (Fig. 3C) and its dissociation from the $\mathrm{APC} / \mathrm{C}$, as shown by $\mathrm{Cdc} 27$ immunoprecipitation experiments from $\mathrm{ex}$ tract and embryos supplemented with $\mathrm{CycB}^{\Delta 90}$ (Fig. 3, D and G). Thus, Cdk1 negatively regulates XErp1's APC/C-inhibitory function.

An additional corollary of our model is that PP2A activity is required to antagonize $\mathrm{Cdk} 1$. In fact, treatment of embryo extract with $1 \mu \mathrm{M}$ of the PP2A inhibitor okadaic acid (OA) quickly induced the hyperphosphorylation of XErpl and the destruction of cyclin $\mathrm{B} 2$ and ${ }^{35} \mathrm{~S}$-labeled securin (Fig. 3E). As shown by Cdc27 immunoprecipitation experiments, PP2A inhibition in embryos or extract resulted in the dissociation of XErpl from the APC/C (Fig. 3, F and G). Notably, prolonged $\mathrm{PP} 2 \mathrm{~A}$ inhibition by $\mathrm{OA}$ induced the destabilization of XErpl in embryos and extract (fig. S3E). Although this mechanism seems not to be relevant for rapid pre-MBT divisions where XErpl levels remain constant (fig. S3A), this observation suggests that $\mathrm{Cdkl}$ in the absence of PP2A activity can affect the turnover of XErp1. In Xenopus egg extract, the PP2A holoenzyme is targeted to XErpl via the B'56 subtype of regulatory subunits (15). To test whether the same applies for embryonic divisions, we performed immunoprecipitation experiments from embryo extract expressing FLAG-tagged B'56e. Endogenous XErp1 efficiently coimmunoprecipitated with FLAG-B'56e but not with FLAG-B55 $\delta$ (Fig. $3 \mathrm{H}$ ). XErpl residues $\mathrm{Ser}^{335}, \mathrm{Thr}^{336}, \mathrm{Ser}^{342}$, and $\mathrm{Ser}^{344}\left(\mathrm{STS}_{2}\right)$ are critical for $\mathrm{PP} 2 \mathrm{~A}$ recruitment in meiosis $(14,15)$. To test whether these residues are also critical for the mitotic interaction between XErpl and PP2A-B'56, we performed pull-down experiments from embryo extract using
A

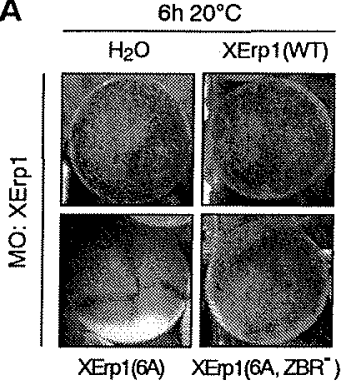

B

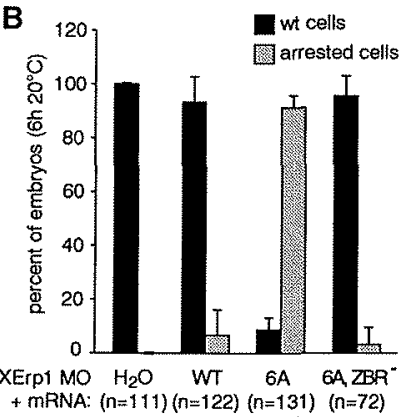

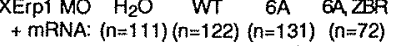

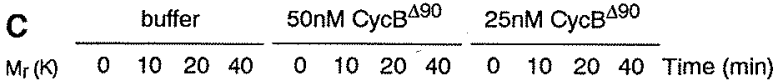
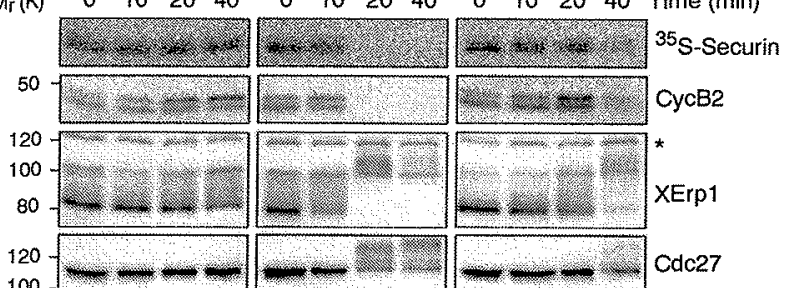

Fig. 3. XErp1 is regulated by phosphorylation. (A and $\mathbf{B}$ ) One-cell embryos were injected with XErp1-MO and $\mathrm{H}_{2} \mathrm{O}$ or mRNA encoding WT, Cdk1 phosphomutant $\mathrm{ST}_{5} \rightarrow 6 \mathrm{~A}(6 \mathrm{~A})$, or Cdk1 phosphomutant XErp1 defective in $A P C / C$-inhibition $(6 A, Z B R)$. At 6 hpf, images were taken (A) and phenotypes quantified (B). (C) Embryo extract was supplemented with nondegradable cyclin $B\left(C y c B^{\Delta 90}\right)$ and analyzed by immunoblot. Time course started with $C y c B^{\Delta 90}$ addition. (D) Cdc27 was immunoprecipitated from extracts treated as in (C) and supplemented with MG-262. Samples were CIP-treated before immunoblotting for $\mathrm{Cdc} 27$ and XErp1. (E) Embryo extract was supplemented with $1 \mu \mathrm{M} \mathrm{OA}$ or dimethyt sulfoxide (DMSO) as solvent control, and samples were CIP-treated as indicated and analyzed as in (C). (F) From extracts treated as in $(E)$ and supplemented with MG-262, Cdc27 was immunoprecipitated. Samples were CIP-treated and immunoblotted for XErp1 and Cdc27. (G) One-cell embryos were injected with $50 \mathrm{nM}$
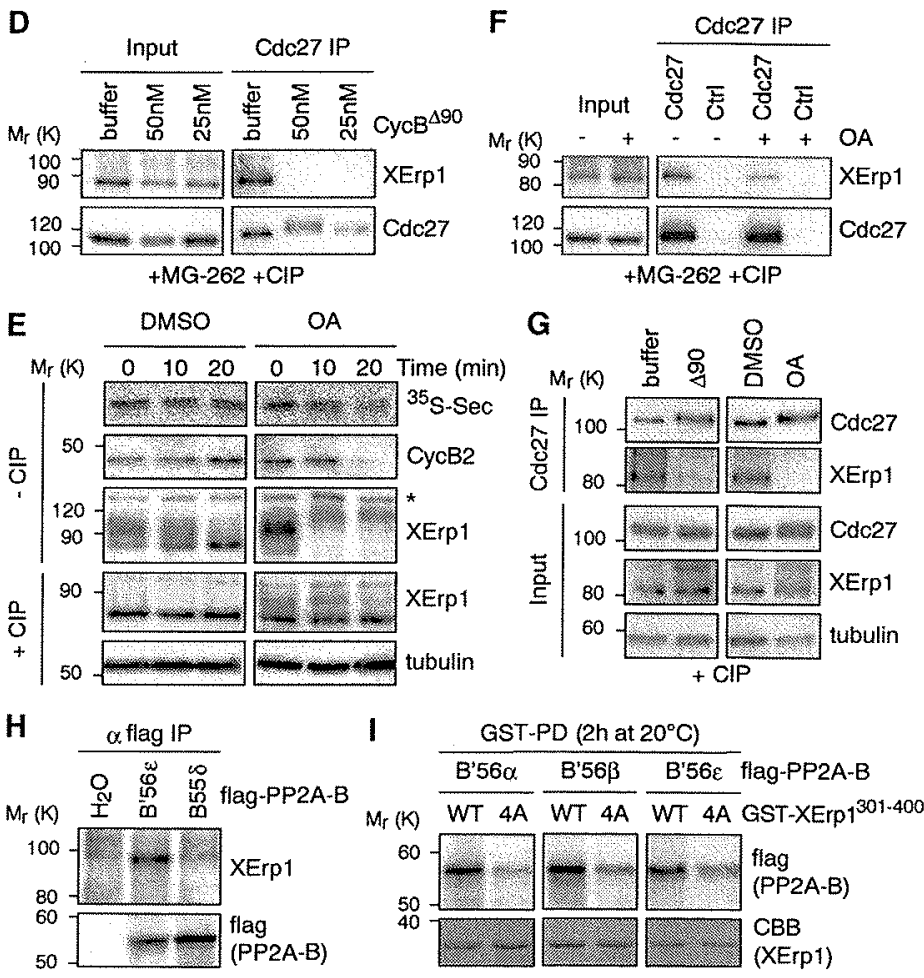

$\mathrm{CyCB}{ }^{\Delta 90}$ or incubated in $2 \mu M$ OA for $30 \mathrm{~min}$. $\mathrm{CdC} 27$ immunoprecipitates were $\mathrm{ClP}$. treated and analyzed by immunoblot. $(\boldsymbol{H})$ mRNA encoding FLAG-tagged PP2A B subunits or $\mathrm{H}_{2} \mathrm{O}$ (control) was incubated in embryo extract and immunoprecipitated using antibodies to FLAG. Precipitates were analyzed for XErp1 and FLAG epitope. (I) Extracts were incubated with B'56 mRNAs and GST-tagged XErp1 ${ }^{301-400}$ WT or PKA phosphomutant (4A) XErp1. After reisolation of GST-tagged proteins, interacting $B^{\prime} 56$ subunits were analyzed by immunoblot. Inputs $[(H)$ and $(I)]$ are shown in fig. $\$ 3, \mathrm{~F}$ and $\mathrm{G}$. CBB, Coomassie Brilliant Blue. 
glutathione $S$-transferase (GST)-tagged WT and mutant $\left(\mathrm{STS}_{2} \rightarrow 4 \mathrm{~A}\right.$ ) XErp1 fragments (residues 301 to 400 ). As shown in Fig. 3I, FLAG-B'56 $\alpha$, FLAG-B'56ß, and FLAG-B'56 $\varepsilon$ efficiently associated with the WT fragment but not the $4 A$ fragment.

Fertilization triggers destruction of cMos (I7) the upstream kinase of the MAPK-p90Rsk pathway - which raises a question about which kinase is critical for XErpl activity in mitosis. XErpl residues $\mathrm{Ser}^{335}, \mathrm{Thr}^{336}, \mathrm{Ser}^{342}$, and $\mathrm{Ser}^{344}$, which are critical for PP2A-B'56 recruitment (Fig. 31 ), are flanked by highly conserved residues matching the protein kinase $\mathrm{A}(\mathrm{PKA})$ consensus phosphorylation motif (18) (fig. S4A). To test whether XErpl is a substrate of PKA, we performed in vitro assays using full-length XErpl fused to maltose-binding protein (MBP). MBPXErp ${ }^{\text {WT }}$ but not MBP-XErp $1^{4 A}$ was efficiently phosphorylated by PKA in vitro (Fig. 4A). To analyze whether XErpl phosphorylation in embryos is mediated by PKA, we incubated MBP. XErpl ${ }^{\mathrm{BD}}$ (residues 319 to 375 ) in $\left[\gamma-{ }^{32}\right.$ P]adenosine triphosphate (ATP)-supplemented control or PKAdepleted embryo extract (Fig. 4B). The WT but not the $4 \mathrm{~A}$ mutant was efficiently phosphorylated in control-depleted extract (Fig. 4C). PKA depletion diminished MBP-XErp $1^{\mathrm{BD} \text {,WT }}$ phosphorylation, which was specific for PKA deple- tion (Fig. 4C). Thus, PKA phosphorylates XErp1 at sites relevant for PP2A-B'56 binding (see Fig. 31), which suggests that PKA might promote the recruitment of PP2A to XErpl.

To test this idea, we incubated in vitro PKAphosphorylated MBP-XErpl ${ }^{\mathrm{BD}}$ in embryo extract and analyzed PP2A levels associated with reisolated MBP-XErpl ${ }^{\mathrm{BD}}$. Under stringent conditions where PP2A did not associate with unphosphorylated MBP-XErpl ${ }^{\mathrm{BD} . W T}$, prephosphorylation of MBP-XEm $1^{\mathrm{BD}, W \mathrm{~T}}$ but not of XErpl ${ }^{\mathrm{BD}, 4 \mathrm{~A}}$ drastically increased the amount of copurified PP2A (Fig. 4D). Consequently, expression of full-length myc-XErpl ${ }^{4 A}$ failed to rescue loss of endogenous XErpl in embryos (Fig. 4E and fig. S4, B and C). Expression of full-length myc-XErpl mutated at either motif $\mathrm{Ser}^{335} / \mathrm{Tht}^{336}$ (S335/T336A) or $\mathrm{Ser}^{342} / \mathrm{Ser}^{344}(\mathrm{~S} 342 / \mathrm{S} 344 \mathrm{~A})$ was also inefficient in complementing loss of endogenous XErpl, and the corresponding MBP-XErp ${ }^{\mathrm{BD}}$ fragments were less efficiently phosphorylated by PKA in vitro than were MBP-XErp ${ }^{\mathrm{BD} \text {,WT }}$ fragments (fig. S4, $D$ to G). Thus, although $\mathrm{Ser}^{342} / \mathrm{Ser}^{344}$ does not match the PKA consensus motif, our data-in line with previous reports (II)-suggest that phosphorylation of both motifs is important for the APC/C-inhibitory function of XErpl. Finally, we tested the prediction that PKA inhibition should result in a phenotype reminiscent of that of XErpl- depleted embryos. To this end, we analyzed embryos treated with the PKA inhibitors $\mathrm{H} 89$ or PKI $(19,20)$. At 24 hpf, the majority of PKAinhibited embryos failed to undergo blastopore closure and died (Fig. 4F and fig. S4H). Immunoblot analyses confirmed efficient PKA inhibition in these embryos (Fig. 4G). Prolonged PKA inhibition - like that of PP2A (fig. S3E)resulted in reduced levels of XErpl (Fig. 4G), further confirming our idea that PKA, via PP2A-B'56, antagonizes Cdkl's negative effect on XErpl in mitosis.

Our studies identify XErpl as a mitotic APC/C inhibitor essential for the fast embryonic preMBT divisions. According to our model (Fig. 4H) $\mathrm{XErp}$ 1-mediated $\mathrm{APC} / \mathrm{C}$ inhibition allows cyclin $B$ accumulation and thus entry into mitosis. At early mitosis, Cdkl's inhibitory effect on XErpl is efficiently antagonized by PP2A-B'56. Once Cdkl reaches maximal activity, it prevails over $\mathrm{PP} 2 \mathrm{~A}$ and thereby triggers $\mathrm{APC} / \mathrm{C}$ activation. During mitotic exit, XErpl must remain inactive despite decreasing Cdkl activity, which suggests the existence of a molecular switch that inactivates PP2A-B'56 and/or PKA at anaphase. In embryo extract, PKA activity remained constant during early divisions (fig. S4I), indicating that PP2A-B'56 might be the target of such a mechanism. At mitotic entry, Cdk1 promotes inactivation of its
A

B

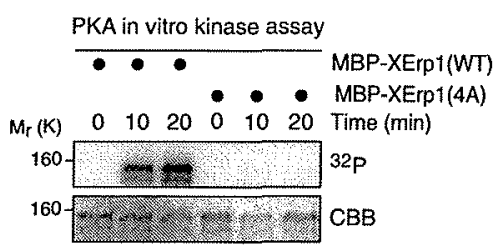

C

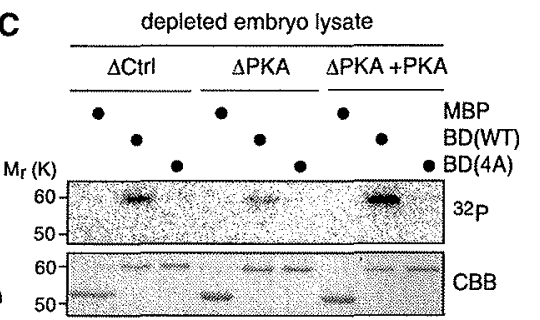

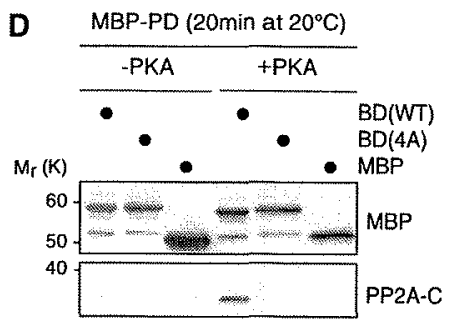
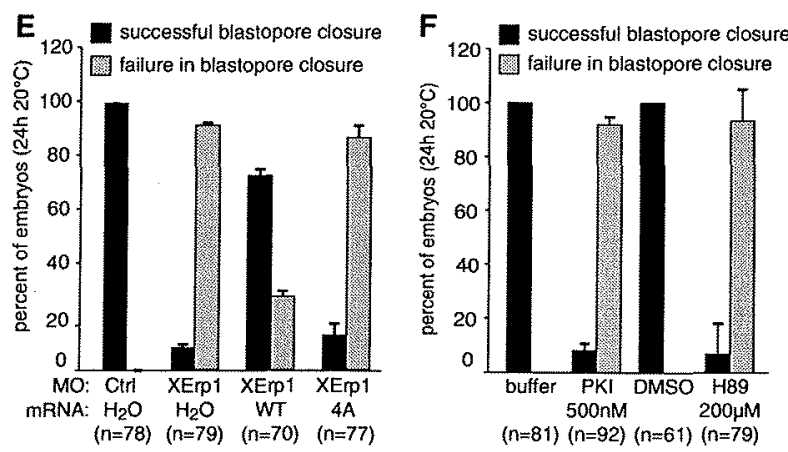

G

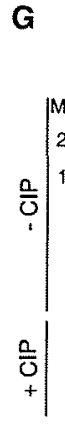

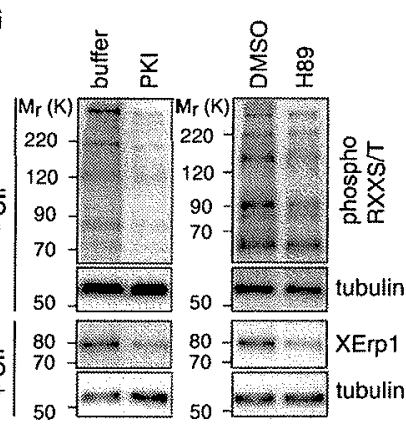

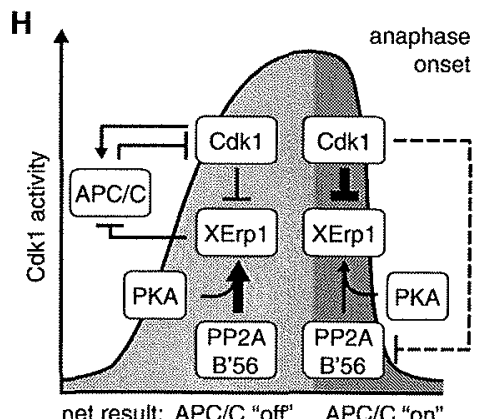

Fig. 4. PKA phosphorylates $X E r p 1$ at sites critical for $P P 2 A B^{\prime} 56$-recruitment. (A) In vitro PKA phosphorylation assay using $\left[\gamma^{-}{ }^{32} P\right] A T P$ and $M B P$-tagged fulllength WT or PKA phosphomutant (4A) XErp1. $\gamma^{32} \mathrm{P}$ incorporation was analyzed by autoradiography. (B) Embryo lysate depleted of PKA $(\triangle P K A)$ or treated with control antibodies $(\triangle \mathrm{Ctrl})$ were immunoblotted for PKA and tubulin. (C) Embryo lysates from (B) were supplemented with $\left[\gamma^{-32}\right.$ P]ATP and phosphorylation of MBP-XErp1 ${ }^{B D}$ was analyzed by autoradiography. Recombinant PKA was added to $\triangle$ PKA lysate. (D) MBP-XErp1 ${ }^{\mathrm{BD}}$ was phosphorylated by PKA in vitro and incubated for $20 \mathrm{~min}$ in embryo extract. After repurification of MBP-XErp1 ${ }^{\mathrm{BD}}$, the associated PP2A catalytic subunit (PP2A-C) was analyzed by

immunoblot. (E) One-cell embryos were injected with XErp1-MO or control MO and $\mathrm{H}_{2} \mathrm{O}$ or mRNA encoding WT or PKA phosphomutant (4A) XErp1; at $24 \mathrm{hpf}$, the indicated phenotypes were quantified. Images and immunoblots are shown in fig. S4, B and C. (F) One-cell embryos were incubated with $\mathrm{H} 89$ or injected with PKI to inhibit PKA; at $24 \mathrm{hpf}$, the indicated phenotypes were quantified. Images are shown in fig. S4H. (G) Embryos were treated as in (F); samples were taken at 6 hpf, CIP-treated as indicated, and immunoblotted for XErp1 and the phosphorylated PKA consensus motif (RXXpS/pT, where $X$ stands for any amino acid and $\mathrm{pS} / \mathrm{pT}$ for phosphorylated serine or threonine) to monitor PKA activity. (H) Model of APCIC regulation in early mitotic divisions. 
antagonist PP2A-B55 8 via the Gwl-Ensa-Arpp19 pathway $(2 l-23)$. Perhaps Cdkl itself could inactivate PP2A-B'56 once it reaches maximal activity in metaphase. By identifying XErpl as a mitotic APC/C inhibitor, our studies provide a framework for understanding how different regulatory modules composed of phosphorylations of Cdc20, APC/C, XErpl, and possibly PP2A$B^{\prime} 56$ are interconnected to create oscillatory $\mathrm{Cdk} 1$ activity driving cell cycle progression.

References and Notes

T. Duncan, T. T. Su, Curr. Biol. 14, R305 (2004)

2. R. S. Hartley, R. E. Rempel, J. L. Maller, Dev. Biol. 173. 408 (1996).

3. ). L. Maller et al, cell cycle transitions in early Xenopus development. Novartis Found. Symp. 237, 58 (2001)

4. ]. E. Ferrell Jr., M. Wu, ]. C. Gerhart, G. S. Martin Mol. Cell. Biol. 11, 1965 (1991).

5. P. Clute, Y. Masui, Dev. Growth Differ, 34, 27 (1992).
6. K. Ohsumi, A. Koyanagi, T. M. Yamamoto, T. Gotoh, Tishimoto, Proc. Natl. Acad. Sci. U.S.A. 101, 12531 (2004).

7. A. Schmidt et al., Genes Dev. 19, 502 (2005).

8. D. Inoue, M. Ohe, Y. Kanemori, T. Nobui, N. Sagata Nature 446, 1100 (2007)

9. J. Lia, B. Grimison, A. L. Lewellyn, J. L. Maller, 1. Biol. Chem. 281, 34736 (2006)

10. ]. Liu, ]. L. Maller, Curr, Biol. 15, 1458 (2005)

11. T. Nishiyama, K. Ohsumi, T. Kishimoto, Nature 446 . 1096 (2007).

12. N. R. Rauh, A. Schmidt, ]. Bormann, E. A. Nigg, T. U. Mayer, Nature 437, 1048 (2005).

13. See supplementary materials on Science Online.

14. J. Q. Wu et al., Proc. Natt. Acad. Sci. U.S.A. 104, 16564 (2007).

15. M. Isoda et al., Dev. Cell 21, 506 (2011)

16. Q. Wu et al., Curr. Biol. 17, 213 (2007).

17. N. Sagata, N. Watanabe, G. F. Vande Woude, Y. lkawa Nature 342, 512 (1989).

18. C. M. Smith, E. Radzio-Andzelm, P. Madhusudan, P. Akamine, S. S. Taylor, Prog. Biophys. Mol, Biol. 71 313 (1999).

19. C. D. Ashby, D. A. Walsh, J. Biot. Chem. 248, 1255 (1973).

20. T. Chijiwa et al., J. Biol. Chem. 265, 5267 (1990).
21. A. Gharbi-Ayachi et al., Science 330, 1673 (2010). 22. S. Mochida, S. L. Masten, M. Skehel, I. Hunt, Science $330,1670(2010)$

23. J. Yu, Y. Zhao, Z. Li, S. Galas, M. L. Goldberg, Mol. Cell 22, 83 (2006).

Acknowledgments: We thank D. Bertinetti, A. Bürkle, J. Gannon, T. Hunt, F. W. Herberg, I. Lorca, S. Mochida, and N. Sagata for reagents; the team of D. Schopper and G. Mende for excellent animal care; and the Mayer group and $B$. Novak for discussions. The authors declare no competing financial interests. Supported by the Doc-fForte program of the Austrian Academy of Science and by the Collaborative Research Center 969 of the German Research Foundation (DFG). 